\title{
Primary Synovial Osteochondromatosis of Knee (Reichel-Jones- Henderson Syndrome): A Radiological Case Report
}

\author{
Zohaib Hussain ${ }^{1}$, Parul Dutta ${ }^{2}$ \\ ${ }^{1}$ Post Graduate Resident, Department of Radiology, ${ }^{2}$ Professor \& HOD, Department of Radiology, Gauhati Medical College \\ and Hospital, India
}

Corresponding author: Dr. Zohaib Hussain, House-21, Red Cross Hospital Road, Navagiri, Chandmari, Guwahati, Assam, India

DOI: http://dx.doi.org/10.21276/ijcmsr.2020.5.2.24

How to cite this article: Zohaib Hussain, Parul Dutta. Primary synovial osteochondromatosis of knee (reicheljones-henderson syndrome): a radiological case report. International Journal of Contemporary Medicine Surgery and Radiology. 2020;5(2):B98-B100.

\section{A B S T R A C T}

Introduction: Synovial osteochondromatosis also known as synovial chondromatosis is a form of non-cancerous tumor that develops from the intima of a joint's synovial membrane. The knee is most frequently impacted, with hip joint, small joints of the hand and wrist in descending order. The symptoms and signs of synovial articular osteochondromatosis are ambiguous and radiological evaluation is inevitably required for diagnosis.

Case Report: A 20-year-old male patient presented with gradual onset pain, swelling and restriction of movement of the right knee. Radiological evaluation revealed several intraarticular lesions of similar size and form, scattered across the joint cavity, with characteristic chondroid mineralization of the "ring-and-arc" type. Typical pathognomonic appearance resulted in its diagnosis as a case of primary synovial osteochondromatosis. Arthroscopic surgery was done and the intra articular fragments were removed.

Conclusion: The primary synovial osteochondromatosis (Reichel-Jones-Henderson syndrome) is comprised of hyaline cartilage nodules in the articular, tendon sheath, or bursal sub-synovial tissue. The radiological findings are often pathognomonic and not requiring histological confirmation. Familiarity with these features help in the diagnosis and prompt management of this benign yet rare entity.

Keywords: Synovial, Chondromatosis, Osteochondromatosis, Knee, Radiology, Intra-articular, Loose Bodies, Reichel Syndrome

\section{INTRODUCTION}

Synovial osteochondromatosis also known as synovial chondromatosis is a form of non-cancerous tumor that develops from the intima of a joint's synovial membrane. ${ }^{1,2}$ The knee is most frequently impacted, with hip joint, small joints of the hand and wrist in descending order. The elbow and shoulder joints are least frequently affected; however, any joint may be involved. ${ }^{3}$ The lesions arise as small cartilaginous nodules. The cartilage foci turn pedunculated and can be sequestrated to the synovial cavity to create several loose bodies, frequently in pearly clumps that mimic "snowstorm knee". Many lesions may not be bigger than a rice grain. Synovial osteochondromatosis occurs most commonly in adults, aged 20 through 50 years, with male prevalence. The cartilage foci in chondromatosis are transformed into chondrocytes by metaplasia of the synovial cells. It is unclear what triggers this metaplasia. It has been proposed that the cause lies in the overactivity of embryonic cell rest at the junction of synovium and cartilage. ${ }^{4}$

The symptoms and signs of synovial articular osteochondromatosis are ambiguous. In the knee, the range of mobility can be restricted, inflammation, crepitus and sometimes loose bodies can be felt. Accurate clinical assessment isn't necessarily possible in other involved joints.

\section{Classification}

Milgram identified three stages of synovial osteochondromatosis.

1. Early: No loose bodies, active synovial disorder.

2. Transitional: active synovial disorder and loose bodies.

3. Late: Loose bodies only, without synovial disorder. ${ }^{5}$

\section{CASE REPORT}

A 20 years old male patient, student by occupation and a local resident presented to the Out Patient Department at our institution.

Chief complaints: Gradual onset pain, swelling and restriction of movement of the right knee joint for 1 year. No previous history of: trauma/fever/infection/tuberculosis, etc. There was no previous history of any relevant surgical procedures. The patient is not an athlete.

\section{On examination}

The patient was alert, cooperative and afebrile. Pulse:76 beats/min and BP was 118/84 $\mathrm{mm} \mathrm{Hg}$. 
General examination was within normal limits.

Local examination of the right knee joint revealed diffuse swelling and mild tenderness of the right knee joint. Consequently, the patient was advised further radiological investigations of the right knee joint.

\section{Radiological Evaluation}

Plain X-ray Radiograph: Plain X-ray Radiograph of right knee AP and Lateral views reveal multiple calcifications in and around the right knee joint including in the suprapatellar region, few with typical ring-and-arc appearance of chondroid tissue. These features are suggestive of multiple intra articular loose bodies. (Fig-1)

Computed Tomography (CT): Corresponding coronal and sagittal plain CT images confirm the presence of multiple intra-articular calcified loose bodies. (Fig-2)

Magnetic Resonance (MR) Imaging: Multiplanar PD (proton density weighted) and PDFS (proton density fat saturated) MR images reveal multiple hetero-intense nodular lesions with evidence of effusion in the knee joint cavity and supra-patellar recess. Few of the nodules show internal areas of high PD and PDFS signal intensity, suggestive of lack of mineralization. There is also surrounding myofascial edema. (Fig.3)

Sagittal \& Axial T1W fat saturated post contrast images reveal synovial enhancement of the knee joint with mild peripheral enhancement of the intra-articular nodules, typical pattern of hyaline cartilage enhancement. There is also evidence of synovitis. (Fig.4)

Diagnosis: Based on clinical history with multi-modality
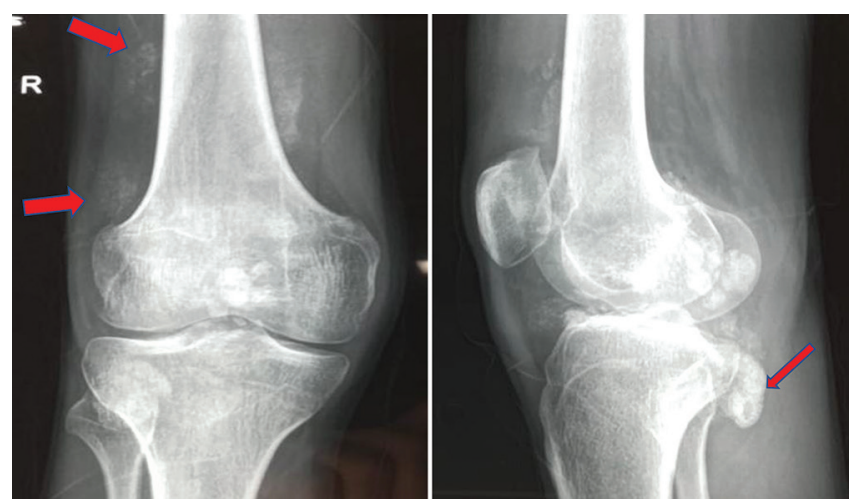

Figure-1: Plain X-ray Radiograph of right knee AP and Lateral at initial presentation
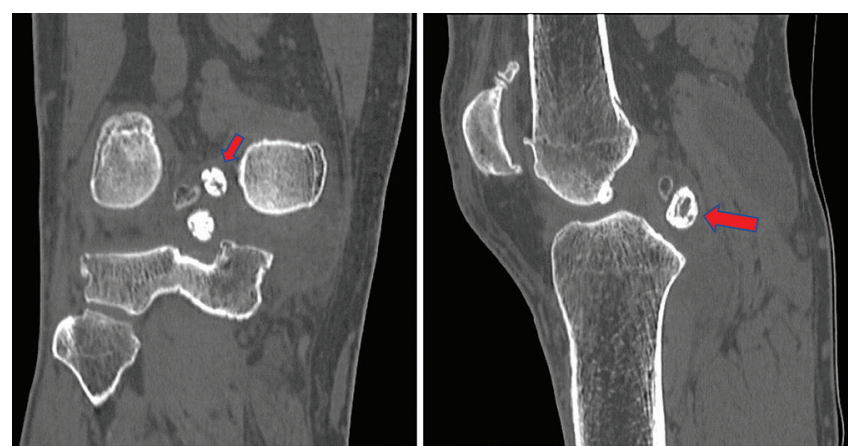

Figure-2: Plain CT images show multiple intra articular loose bodies (arrows) (bone window) imaging findings, a diagnosis of primary synovial osteochondromatosis of the right knee joint (transitional stage as per Milgram ${ }^{5}$ ) was made. The imaging findings are pathognomonic. There is no evidence of any bone erosions, ruling out malignant transformation. There is also evidence of joint effusion and synovitis.

Treatment: Patient subsequently underwent surgery via arthroscopic technique. On surgery, the radiological findings were confirmed with the presence of multiple intra articular loose bodies in the joint cavity and supra patellar recess. These loose bodies were removed. (Fig.5) Rest of the knee joint structures including ACL, PCL and menisci were found to be intact.

Post-operative follow up: The patient was doing fine without any symptoms. The patient was followed up with plain X-ray radiograph of the right knee joint after 3 months. Post-
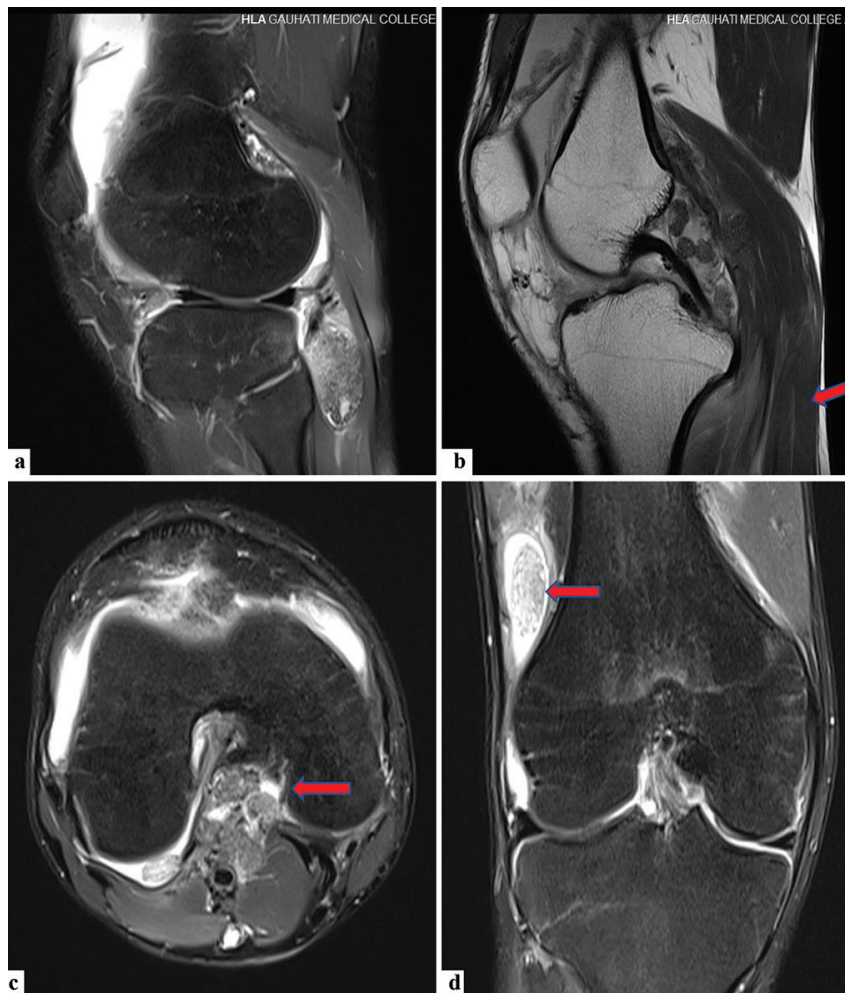

Figure-3: a. Sagittal PDFS, b. sagittal PD, c. axial and d. coronal PDFS MR images showing the intra articular loose bodies with joint effusion.

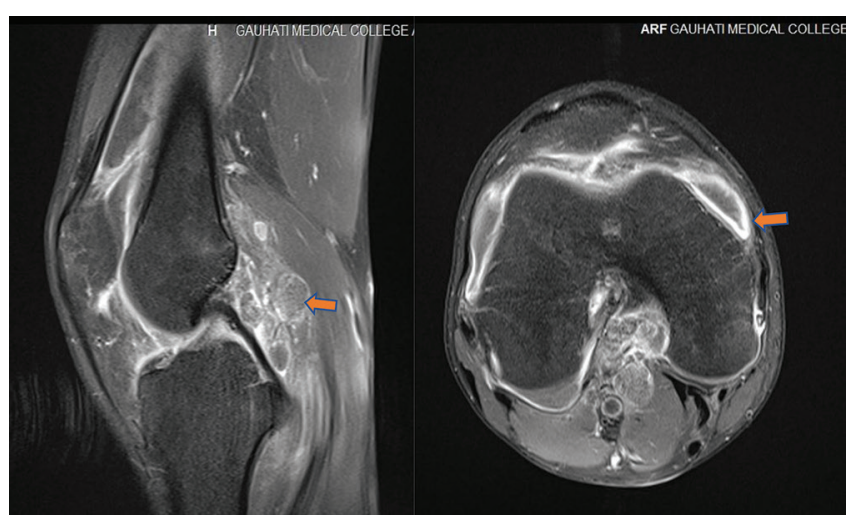

Figure-4: Sagittal \& Axial T1FS Post Contrast MR images 


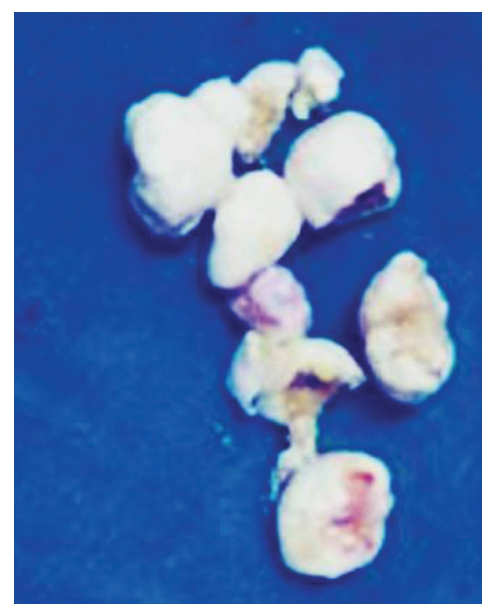

Figure-5: Post-surgical specimen of loose bodies

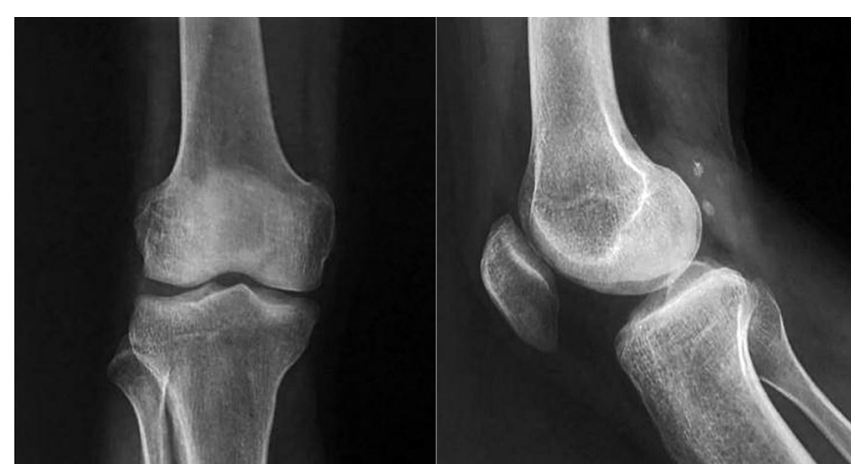

Figure-6: Post-operative plain $\mathrm{x}$-ray radiograph of the knee joint reveals no significant abnormalities.

operative plain $\mathrm{x}$-ray radiograph of the knee joint reveals no significant abnormalities. (Fig-6)

\section{DISCUSSION}

Primary synovial osteochondromatosis describes an abnormal benign neoplastic condition in the sub-synovial area of a bone, joints, tendon sheaths, or bursae with hyaline cartilage nodules. These may be swollen and isolated from the synovium. The knee, followed by the hip joint, is the most frequently affected location and patient demographic of male adults being most common. Because of substantial histological atypia, the pathological presentation can mimic chondrosarcoma, so radiological distinction is essential for appropriate diagnosis to localize the disease as synovial related. ${ }^{6,7}$ The radiological observations are often pathognomonic. Radiographs show several intraarticular lesions $(70 \%-95 \%$ of cases) of similar size and form, scattered across the joint cavity, with characteristic chondroid mineralization of the "ring-and-arc" type. Extrinsic bone erosion happens in $20 \%-50 \%$ of patients. The calcified intra articular fragments and extrinsic bone changes are optimally represented by computed tomography. Magnetic resonance (MR) imaging results are more complex based on the extent of mineralization, but the most typical form (77\% of instances) shows low to moderate T1-weighted signal and very high T2-weighted signal intensities and hypo intense calcifications. Such patterns of signal intensity on MR images and low attenuation of the non-mineralized areas on CT images indicate the increased water content of cartilaginous nodules. ${ }^{8,9}$ Primary disease treatment is surgical synovectomy in which chondral fragments are removed; relapse rates vary from $3 \%$ to $23 \%$. Malignant transition to chondrosarcoma is rare (5 percent of cases), and while challenging to discern from benign disorder, frequent recurrences and invasion of the marrow are suggestive. ${ }^{10}$

\section{CONCLUSION}

The primary synovial osteochondromatosis (Reichel-JonesHenderson syndrome) is comprised of hyaline cartilage nodules in the articular, tendon sheath, or bursal sub-synovial tissue. The radiological findings are often pathognomonic and familiarity with these help in their diagnosis and prompt management. Their non-association with intrinsic joint defect (typically osteoarthritis) and the existence of greater number of chondral bodies separates it from secondary disorder.

\section{ACKNOWLEDGEMENT}

The authors would like to express their gratitude towards the faculties, students and technical staff of the Department of Radiology, GMCH.

\section{REFERENCES}

1. Barnett CH, Davies DV, Mac Conaill MA. Synovial joints. London: Longmans Green 1961.

2. Jaffe HL. Tumors and tumorous conditions of the bones and joints. Philadelphia: Lea and Febiger 1959.

3. Mcivor PR, King DE. Osteochondromatosis of the hip joint. Journal of Bone and Joint Surgery 1962;44-A:87.

4. Lloyd-Roberts GC. The role of capsular changes in osteoarthritis of the hip joint. Journal of Bone and Joint Surgery Br 1953;35-B (4):627-42.

5. Milgram JW. The classification of loose bodies in human joints. Clin Orthop 1977; 124:282-91.

6. Crotty JM, Monu JU, Pope TL Jr. Synovial osteochondromatosis. Radiol Clin North Am 1996;34(1): 327-342.

7. Dorfman HD, Czerniak B. Synovial lesions. In:Bone tumors. St Louis, Mo: Mosby, 1998; 1041-1086.

8. Ginaldi S. Computed tomography feature of synovial osteochondromatosis. Skeletal Radiol 1980;5(3): 219222.

9. Blandino A, Salvi L, Chirico G, et al. Synovial osteochondromatosis of the ankle: MR findings. Clin Imaging 1992; 16(6):34-36.

10. Villacin AB, Brigham LN, Bullough PG. Primary and secondary synovial chondrometaplasia: histopathologic and clinicoradiologic differences. Hum Pathol 1979; 10(3):439-451.

\section{Source of Support: Nil; Conflict of Interest: None}

Submitted: 15-04-2020; Accepted: 17-05-2020; Published online: 26-06-2020 